\title{
Astrometric study of MACHO halo distribution in our Galaxy
}

\author{
Kouji Ohnishi $^{1}$, Mizuhiko Hosokawa ${ }^{2}$, \& Toshio Fukushima ${ }^{3}$ \\ 1 Nagano National College of Technology, Nagano, 381-8550, Japan \\ 2 Communications Research Laboratory, Tokyo, 184-8795, Japan \\ 3 National Astronomical Observatory, Mitaka, Tokyo, 181-8588, Japan
}

\begin{abstract}
We estimated the optical depth and event rate of $\mu$ as level astrometric microlensing for the stars outside our galaxy caused by MACHOs. For the stars in the LMC and the SMC, the optical depth of a $1 \mu$ as detection threshold is on the order of $10^{-1}$ and the event rate of the induced proper motion of $1 \mu \mathrm{as} /$ year is on the order of $10^{-3} / \mathrm{yr}$ with the event duration of around a hundred years. They depend on the distribution of lenses and sources. This poses a constraint on the expected probability of photometric self-lensing in LMC and SMC.
\end{abstract}

\section{Introduction}

Astrometric microlensing is one of the most promising tools for studying invisible lenses like MACHOs and low-mass stars in the Galaxy. Some observational projects aiming for $10 \mu$ as accuracy or better (e.g. VERA, SIM, \& GAIA) will make it possible to observe the positional shift of the image centroid due to stars and MACHOs in our Galaxy.

We have investigated the astrometric microlensing by the stars and MACHOs in the Galaxy, and have shown the estimation of the degradation of reference frames and some ways to measure the mass distribution around the Galactic center (Hosokawa, Ohnishi \& Fukushima 1997; Hosokawa et al. 2002; Ohnishi, Hosokawa \& Fukushima 2003).

In this paper, we estimate the astrometric microlensing optical depth $\tau_{A}$ and event rate $\Gamma_{A}$ for the stars outside our galaxy by MACHOs. Here we consider the LMC stars and SMC stars as source objects. Comparison of $\tau_{A}$ and $\Gamma_{A}$ with photometric optical depth $\tau_{P}$ and event rate $\Gamma_{P}$ is also discussed.

\section{Event Rate toward the LMC}

For the estimation of $\tau_{A}$ and $\Gamma_{A}$ of the astrometric microlensing toward the LMC, we adopt the model parameters of our galaxy and LMC from Gyuk et al. (2000). The results are shown in Table 1 . Here $f$ and $f_{\mathrm{LMC}}$ are the mass fraction of MACHOs within the dark matter in the Galaxy and LMC. The event rate is about $10^{-3} \mathrm{f} / \mathrm{yr}$ for a $1 \mu \mathrm{as} / \mathrm{yr}$ detection threshold. We see that the average event duration $t_{E}$, defined as $\tau_{A} / \Gamma_{A}$, for the Galactic MACHO lensing is more than 100 years. Similar calculations give us the $\tau_{A}$ and $\Gamma_{A}$ for SMC stars. 
Table 1. Optical Depth $\tau_{A}$ and Event Rate $\Gamma_{A}$ toward the LMC

\begin{tabular}{cc|cccc}
\hline \multicolumn{2}{c|}{ Lens in our Galaxy } & \multicolumn{4}{|c}{ Lens in LMC } \\
\hline$\tau_{\text {halo }}$ & $\Gamma_{\text {halo }}$ & $\tau_{\text {halo }}^{\prime}$ & $\Gamma_{\text {halo }}^{\prime}$ & $\tau_{\text {disk }}^{\prime}{ }^{*}$ & $\Gamma_{\text {disk }}^{\prime}{ }^{*}$ \\
\hline $0.16 f$ & $1.2 \times 10^{-3} f$ & $4 \times 10^{-3} f_{\text {LMC }}$ & $8 \times 10^{-5} f_{l m c}$ & $2 \times 10^{-4}$ & $2 \times 10^{-4}$ \\
\hline
\end{tabular}

Note $-\tau$ and $\Gamma$ are the optical depth in the case of a $1 \mu$ as detection threshold and event rate $\left(\mathrm{yr}^{-1}\right)$ in the case of a $1 \mu \mathrm{as} / \mathrm{yr}$ detection threshold, respectively. $\tau_{\text {halo }}$ and $\Gamma_{\text {halo }}$ are those for Galactic MACHOs, while $\tau_{\text {halo }}^{\prime}$ and $\Gamma_{\text {halo }}^{\prime}$ are those for LMC MACHOs. $\tau_{\text {disk }}^{\prime}{ }^{*}$ and $\Gamma_{\text {disk }}^{\prime}{ }^{*}$ are calculated for the direction toward the LMC center. The contribution of the Galactic disk stars to the optical depth and the event rate are estimated to be much smaller than $\tau_{\text {halo }}$ and $\Gamma_{\text {halo }}$.

\section{Discussion}

We found that $\tau_{\text {halo }}$, i.e. $\tau_{A}$ for the Galactic MACHO halo, is much larger than that for any other components. On the contrary, $\tau_{P}$ for the Galactic MACHO halo is comparable to that of the LMC disk stars (Gyuk et al. 2000). This is because the most effective position of the lens is near the observer in the former case and around the mid point in the latter. As for the event rate, if $f$ is smaller than $0.2, \Gamma_{A}$ for Galactic MACHOs and for LMC disk stars are comparable. However, the time scale for LMC disk stars is much shorter than that for Galactic MACHOs, about 1 year, because of the smallness of the maximum positional shift of LMC disk stars, about $1 \mu \mathrm{as}$. Thus we cannot detect any self-lensing events with the accuracy of $2-3 \mu$ as astrometry. This is also different from the case of photometric lensing. Therefore, comparing the astrometric microlensing observation with the photometric one, we can judge the mean distance to the lens. This will give us an answer to the self-lensing hypothesis on the result of the MACHO collaboration (Alcock et al. 2000).

We estimated $\tau_{A}$ and $\Gamma_{A}$ for the LMC and the SMC using the flattened halo model. Comparing these event rates, the flattened parameter of the MACHO halo can be determined. $\Gamma_{S M C} / \Gamma_{L M C}$ is about 0.80 for the spherical halo, and 1.05 for an E6 halo, independent of other parameters.

\section{References}

Alcock, C. et al. 2000 ApJ, 542, 281

Gyuk, G., Dalal, N., Griest, K. 2000, ApJ, 535, 90

Hosokawa, M., Ohnishi, K., \& Fukushima, T. 1997, AJ, 114, 1508

Hosokawa, M., et al. 2002, ApJ, 580, L43

Ohnishi, K., Hosokawa, M, Fukushima, T. 2003 in ASP Conference Series, Vol.289, IAU 8th Asian-Pacific Regional Meeting, eds. S. Ikeuchi, J. Hearnshaw, and T. Hanawa, 461 